for both lytic growth and transformation.

There are two regions where differences exist among the three strains of BK sequenced. The first is located in the noncoding tandem repetitive sequences to the late side of the origin of DNA replication. There is a 42 base pair deletion in the $\mathrm{BK}$ (Dun) genome compared with the prototype BK sequence. The BK(MM) genome varies more markedly from the other two DNAs in both the sizes and the patterns of these repeated sequences. Tandem repetitive segments are located in the corresponding region of the SV 40 genome although they bear no sequence homology to the repeats of BK DNA. Variation also occurs in these tandem repetitive segements among different strains of SV40 and portions of these repetitive segments can be deleted without any apparent effect on the biological function of the virus. Thus there is apparently little sequence constraint in this region among papovaviruses generally or even among strains of the same virus, and its biological function and significance remain obscure.

Differences were also noted in the early region of the genomes of the BK strains. Although the sequences of the early regions of the prototype $\mathrm{BK}$ and $\mathrm{BK}$ (Dun) are identical, there is a deletion of 262 base pairs in the $\mathrm{BK}(\mathrm{MM})$ early region. This deletion removes sequences from the putative intervening sequence for large $T$ antigen, including the likely donor site for the small $t$ antigen splice junction. As a consequence, BK(MM) does not encode a small t antigen (Seif et al. Cell 18, 963; 1979) making it equivalent to some of the constructed early viable deletion mutants of SV 40 which map in the analogous region of the SV40 genome. Since BK(MM) can replicate in tissue culture and can induce tumors in vivo as well as transform cells in vitro, the small $\mathrm{t}$ antigen must be dispensible for these functions of BK.

While remarkable similarities can also be found between the genome of BK (or SV40) and that of polyoma, significant differences exist (Soeda et al. Nature 283, $445 ; 1980)$. The BK and SV40 genomes contain a large non-coding region between the carboxy terminal ends of large $T$ antigen and VP1 (approximately 100 base pairs), whereas the same region in polyoma contains only seven tase pairs. In addition the polyoma genome does not contain the region of tandem repetitive segments in the non-coding region to the late side of the origin of DNA replication as do $\mathrm{BK}$ and SV40. The late leader regions of each of the BK and SV40 genomes contain an open reading frame following an initiator AUG referred to as the 'agnogene'. Although proteins encoded by these sequences have not yet been detected, approximately $2 / 3$ of the putative amino acids are conserved

Peter M. Howley is in the Laboratory of Pathology, National Cancer Institute, National Institutes of Health, Bethesda, Maryland.

\title{
Testing gravitational theories
}

\section{from Bruno Bertotti}

DURING their mission to Mars in 1976 and 77 the four Viking spacecraft verified Einstein's predictions concerning the additional delay in the propagation of an electromagnetic signal due to the gravitational field of the Sun with an accuracy of $2 \%$, much better than any previous determination. A recent letter to the Astrophysical Journal (234, L219; 1979) by R.D. Reasenberg and others has now made public the details of this outstanding experimental achievement. This additional delay has the largest value, $250 \mu \mathrm{s}$, when the signal grazes the Sun; with the Viking spacecraft (two orbiters and two landers) it was possible to measure corrections of $50 \mathrm{~ns}$, corresponding to distances of $7.5 \mathrm{~m}$. The main technical reason for this large precision was that the signals came from a lander, firmly anchored to the surface of the planet, whose motion is very regular and predictable.

This is another example of the extraordinary accuracy to which space research has pushed our knowledge of the geometry of the Solar System and, consequently, of such dynamical properties as the planetary masses; and there is still scope for improvement, mainly through the implementation of better telecommunication systems at higher frequencies (including the use of laser signals).

In my view this experiment marks also the end of one phase in gravitational research, which aimed at testing all the

Bruno Bertotit is at the Istituto di Fisica Teorica, Universita di Pavia.

between the BK and SV40 genome suggesting perhaps, that a protein exists. No corresponding 'agnogene' is present in the late leader region of polyoma.

The most significant difference between the DNAs of BK(or SV40) and Py lies in the genetic organisation of the early regions and possibly reflects the different host ranges of these viruses. The BK and SV40 early regions are extensively homologous throughout and each encodes only two known major early proteins, large $T$ and small $t$ antigens. The Py early region encodes a third early protein, middle $T$ antigen (Ito et al. Proc. natn. Acad. Sci. U.S.A. 74, 4666; 1977; Hunter et al. Proc. natn. Acad. Sci. U.S.A. 75, 5917; 1978). Whereas the amino acid sequence of the Py small $t$ antigen is remarkably similar to those of BK and SV40, the predicted Py large $T$ antigen differs in two regions significantly from those of BK and SV40. First there is a 130 amino acid segment ('insertion') unique to the Py large $T$ antigen located 100 nucleotides after the theories of gravitation which are based on the assumption that space-time has a metric structure (described by its curvature) and that the Principle of Equivalence - all local laws have a universal validity - holds. These assumptions have been precisely formulated in a mathematical scheme, which has also led to the classification and the experimental consequences of all possible theories of this kind (see for example, Will, in Experimental Gravitation (Ed. Bertotti) Academic Press, New York, 1973). In recent years all these theories have been to a large extent falsified by experiment and theoretical arguments, except general relativity, whose predictions have all been confirmed to a greater and greater accuracy.

It seems, therefore, that the experimental programs within this theoretical framework have passed the point of diminishing returns. This does not mean, however, that in the physics of gravitation there are no open questions and potential anomalies. Perhaps the most important one is the possible change of the constant of gravitation $G$ over a cosmological time scale, which has been extensively studied by P. Dirac and V. Canuto. If this is true, not only is Einstein's theory violated, but the whole of astrophysics and cosmology must undergo a drastic revolution. The tests of the constancy of $G$ are not yet sufficiently precise to exclude this possibility; and precise geometrical measurements in the Solar System, especially if extended over a long period of time, will probably be crucial.

early acceptor splice junction which has no counterpart in BK or SV40. The second difference is the carboxy terminus of the BK and SV40 large T antigens which is essentially absent from the Py large $T$. Interestingly the segment of DNA encoding this unique carboxy terminal portion in the BK and SV40 genomes is open in a second reading frame for approximately 300 nucleotides providing an additional potential coding segment. Putative peptides encoded by this segment of BK and SV40 would be hydrophobic and might thereby be used in associating a protein with a membrane; however, such peptides have not yet been demonstrated.

\section{0 years ago}

The following arrangements have been made at the Royal Institution for the lectures after Easter. Tuesdays: - Prof. Huxley - Two Lectures on Dogs, and the Problems connected with them; Mr. Robert II. Scott, F.R.S. - Four Lectures on Wind and Weather.

From Nature 21, 11 March, 451; 1880. 